\title{
Varus stress MRI in diagnosis of medial meniscal posterior root tear
}

\author{
Ashgan Ali Abdo Qardash¹, Afaf A. Hassan', Hesham A. El kady², Hosam El-Deen G. Mohammed', \\ Mohamed Abdel-Tawab ${ }^{1 *}$ and Nisreen A. A. Mohammed ${ }^{1}$
}

\begin{abstract}
Background: Early and accurate diagnosis of medial meniscal posterior root tear (MMPRT) via magnetic resonance imaging (MRI) is crucial to prevent the development of medial meniscus extrusion (MME) and subsequent osteoarthritic changes. The study aims to assess the role of MRI in diagnosing the medial meniscal posterior root tear (MMPRT) as well as to investigate the additive value of a new MRI technique (varus stress position technique) in measuring the accurate degree of a truly extruded meniscus, during normal daily activities like standing or walking. This prospective study included 70 patients who had an episode of posteromedial knee pain. All patients underwent conventional MRI, whereas only 35 of them underwent MRI with varus stress loading position.

Results: The study included 70 patients, of which 56 were female (80.0\%), with a mean age of $49.1 \pm 8.5$ years. According to duration onset of MMPRT, 39 patients were in the chronic stage (4-12 months), 16 patients were in the acute stage (early $<1$ month), and 15 patients were in the subacute stage (1-3 months). It was found that varus stress position could detect $100 \%$ of the patients (35/35) with positive medial meniscus extrusion (MME) $(\geq 3 \mathrm{~mm})$, whereas the MRI with the traditional position could detect only $62.9 \%$ of the cases $(22 / 35)(P<0.000)$. Regarding the chronicity of MMPRT, a significant increase was observed in the amount of MME in post-varus MRI compared with that in prevarus MRI for each stage (acute, subacute, and chronic) $(P<0.000)$.
\end{abstract}

Conclusion: $\mathrm{MRI}$ is considered a superior imaging modality in diagnosing MMPRT through several characteristic MRI findings. The varus stress position showed a valuable role in assessing the presence and degree of severity of true extruded meniscus using positional varus stress load.

Keywords: Medial meniscus posterior root tear, Medial meniscus extrusion, Magnetic resonance imaging, Varus stress loading

\section{Background}

The meniscus is the most important cushion in the knee [1]. It plays an important biomechanical role in the axial load distribution of knees utilizing hoop strength [2]. Pain and subsequent arthritis can occur if cushioning is lost because of meniscus injury [1]. Menisci may be torn because of either trauma or degeneration as in osteoarthritis wherein an abnormal distribution of force across

\footnotetext{
*Correspondence: dr.m.tawab@gmail.com

1 Department of Diagnostic Radiology, Faculty of Medicine, Assiut

University, Assiut 71515, Egypt

Full list of author information is available at the end of the article
}

the knee joint occurs. Meniscal root tear is a type of meniscal tear, mostly treated as a meniscal radial tear, that occurs within $1 \mathrm{~cm}$ away from the meniscal root attachment or as a root avulsed bony fragment at the meniscal tibial insertion [3].

MMPRT can extend to the posterior meniscal root attachment and occurs particularly in middle-aged or elderly patients who experience a single event of posteromedial painful popping sensation during light activities, such as climbing the stairs and squatting [4]. MRI is well established as the best imaging modality for the evaluation of a patient with suspected meniscal pathology, including the medial meniscal posterior horn root $[5,6]$. 
Meniscal root tears (MRTs) may occur in either acute or chronic form and are often overlooked or ignored during MRI scans. However, as in meniscal radial tears, root tears may have critical biomechanical sequels [7]. Therefore, early identification of a meniscal root tear on MRI is critical, due to the introduction of a new arthroscopic transtibial pullout repair used to repair meniscal root tears and maintain the tibiofemoral cartilage of the knee [2]. Recently, MMPRTs have been extensively investigated in both arthroscopic and MRI studies [8]. With the widespread use of MRI, the diagnosis of meniscal root injuries has been more accurate. All MR pulse sequences should be reviewed, but T2-weighted coronal sections are considered the best sequence in the visualization of the posterior roots [9].

Medial meniscal posterior root lesions can lead to medial meniscus displacement and the formation of a gap defect at the site of root insertion, which is aggravated when the knee is subjected to stress loads [10].

The quality of MR image and the skill of the radiologist are among the important factors in reaching an accurate diagnosis of a meniscal root tear [11]. It is not hard to diagnose the MMPRT if the diagnosis was based on the main distinguishing signs in MRI-plane images along with clinical symptoms. These MRI signs include giraffe neck sign, cleft sign, ghost sign, radial tear sign, and meniscus extrusion [12]. Some authors consider these main signs as direct signs for the diagnosis of MMPRT, with an exception for meniscus extrusion, which is considered an indirect sign of MMPRT [13].

Evaluation of preoperative MME may be important in determining the duration of injury and treatment planning protocol for MMPRT [14]. Therefore, MME could be used as a further guide in the diagnosis of medial meniscal root tear in MRI [15]. The degree of MME can influence the postoperative clinical outcome of the MMPRT and recovery after transtibial pullout repair [14, 16]. MME may progress after MMPRT more quickly than that in osteoarthritic knees with intact posterior roots [14]. On the other hand, the identification of the accurate degree of the truly extruded meniscus in the supine position with non-weight-bearing MRI could be one of the challenges encountered by previous studies [16].

For that reason, the present study aimed to assess the role of MRI in diagnosing MMPRT as well as to introduce a new MRI technique with varus stress position that could achieve a more accurate measurement of the truly extruded meniscus in MMPRT.

\section{Patients and methods}

\section{Study population}

This prospective study included 70 suspected MMPRT patients based on their clinical presentations during the period between February 2019 and August 2020. Institutional review board approval and written informed consent were obtained for this study. The study included 16 patients in the early (acute) stage, 15 patients in the subacute stage, and 39 patients in the chronic stage, who presented with posteromedial knee pain with or without associated popping sounds. Conventional MRI of the affected knee joint was performed in all studied patients. Overall, 35 patients underwent MRI with varus stress position, whereas 26 of them were in acute and subacute stages and subjected to confirmatory gold standard arthroscopy. Those patients who had previous knee surgery and any general contraindication to MRI as any paramagnetic substance or claustrophobia were excluded from the present study. The flowchart of the current study population inclusion process is illustrated in Fig. 1.

\section{MRI technique}

All MRI scans of the knee were performed via a 1.5 Tesla MRI system (Achieva, Philips Medical Systems, Best, The Netherlands) using a knee surface coil. Before the MRI scan, all patients were subjected to standing/weight-bearing anteroposterior plain X-ray of the affected knee joint for osteoarthritis and varus alignment assessment. Conventional MRI position was done for all patients in terms of multiplanar conventional sagittal, coronal, and axial MRI planes and pulse sequences (Table 1). Fast spinecho proton density (FSE PD) weighted sequences with and without fat-saturation (FS) were the mainstay in this study, as they are accurate and sensitive for evaluation of meniscal root tears in particular. The pulse sequences included as following: sagittal PD, T2, and STIR, coronal T2 and FSE PD, and axial T2WI. The field strength, coil, slice thickness, field of view, matrix size, and other imaging parameters used were optimized to increase the signal-to-noise ratio and decrease scan time, thereby decreasing motion artifact. During this scan, the patient was in the supine position and the legs were positioned in complete or nearly complete extension as the affected knee was put in a cylindrical knee coil (Fig. 2a).

\section{Varus stress position}

Out of the 70 patients, 35 of them were in the varus stress position(Fig. 2b), in which one to two thick sandbagsabout $\approx 8 \mathrm{~cm}$ in thickness-was positioned between the inner wall of the knee coil and the inner surface of the other sound knee, and then a band wrapped the lower legs to bind both ankle joints close together. In this new technique, we positioned both knee joints in a varus stress position by causing stress load on the medial compartment of the knee, particularly the medial meniscus, leading to the presence or subsequently increased amount of the extruded meniscus in MMPRT's patients. 


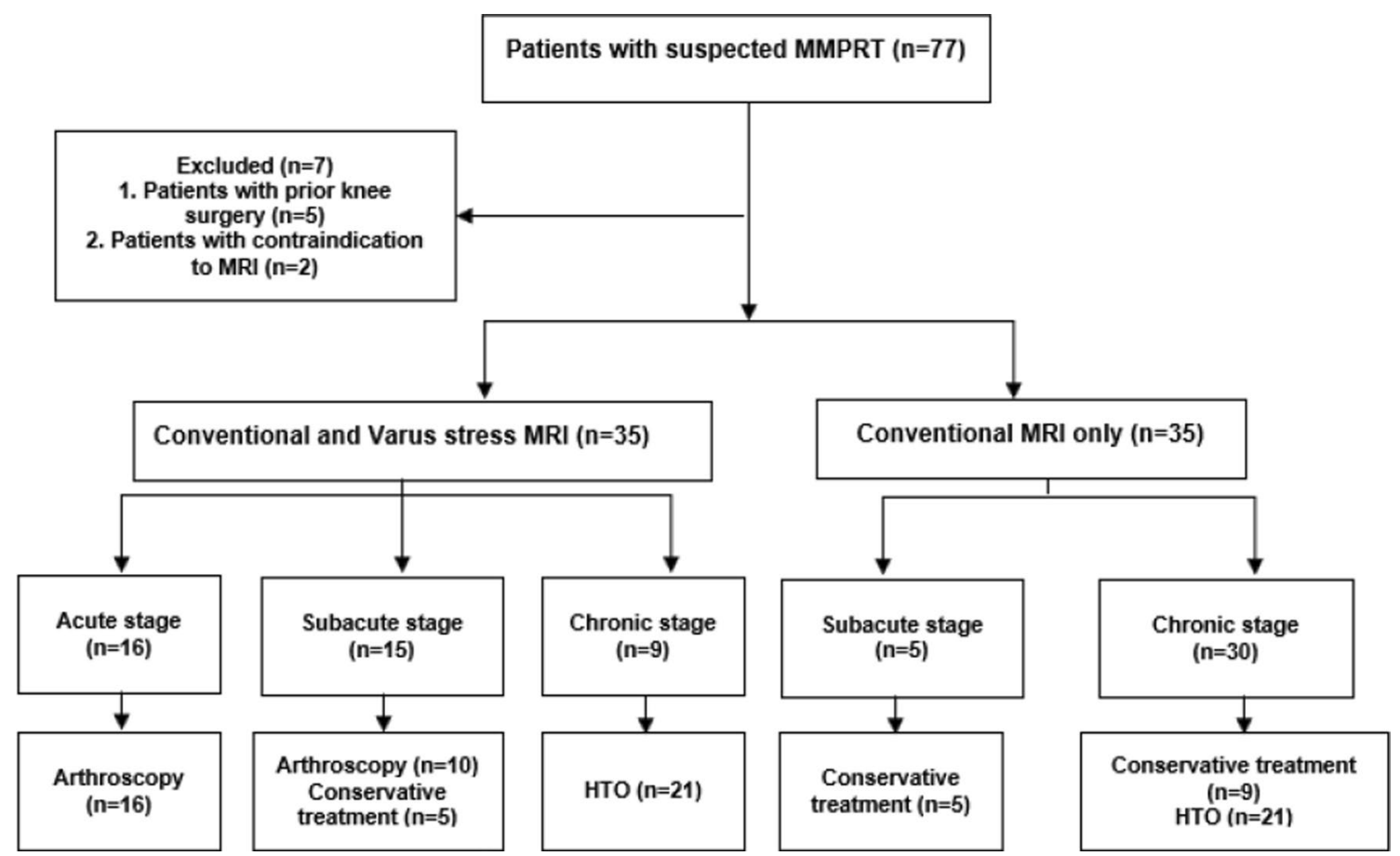

Fig. 1 Flowchart of our study

Table 1 Protocol for MRI examination of knee joint and meniscus

\begin{tabular}{|c|c|c|c|c|c|}
\hline Sequences parameters & $\begin{array}{ll}\text { Sagittal PD } & \text { Sagittal T2 } \\
\text { DUAL_DR_TSE } & \end{array}$ & Sagittal STIR & Coronal T2 & Coronal PD-SPAIR & Axial T2 \\
\hline TR & 2078 & 2728 & 4745 & 3000 & 4861 \\
\hline TE & 110 & 60 & 100 & 30 & 100 \\
\hline \multicolumn{6}{|l|}{$\mathrm{FOV}(\mathrm{mm})$} \\
\hline Anterior/ Posterior(AP) & 150 & 150 & 79 & 79 & 160 \\
\hline Right/left (RL) & 79 & 79 & 160 & 160 & 160 \\
\hline Feet/head (FH) & 150 & 150 & 160 & 160 & 79 \\
\hline$A C Q$ matrix $M \times P$ & $320 \times 240$ & $256 \times 203$ & $324 \times 253$ & $292 \times 240$ & $324 \times 260$ \\
\hline Reconstruction matrix & 512 & 512 & 560 & 512 & 560 \\
\hline Slice thickness (mm) & 3 & 3 & 3 & 3 & 3 \\
\hline Min. Slice gap (mm) & 3 & 3 & 0 & 3 & 0 \\
\hline Flip angle & 90 & 90 & 90 & 90 & 90 \\
\hline Slices & 24 & 20 & 24 & 24 & 24 \\
\hline
\end{tabular}

TR, Repetition time; TE, echo time; FOV, field of view (ranged from 15 to $16 \mathrm{~cm}$ ); DUAL_DR_TSE, double PD and T2 weighted sequences

The average duration time of the examination was from 25 up to $30 \mathrm{~min}$

The additional varus stress coronal T2WI and PD-SPAIR pulse sequences were performed to measure and compare the pre- and post-varus stress MME.

\section{MR image interpretation}

The analysis of MRI was based on several characteristic direct (cleft/truncation sign, giraffe neck sign, ghost meniscus sign, and the radial tear sign) and indirect (medial meniscus extrusion) MRI findings of MMPRT observed on all MRI planes (Fig. 3). In the present study, T2- and PD-SPAIR-weighted images were the essential pulse sequences. On the posterior (the slice that shows the fibular head) and mid-coronal (the slice showing the medial spine of the tibia at its largest area) planes, the 


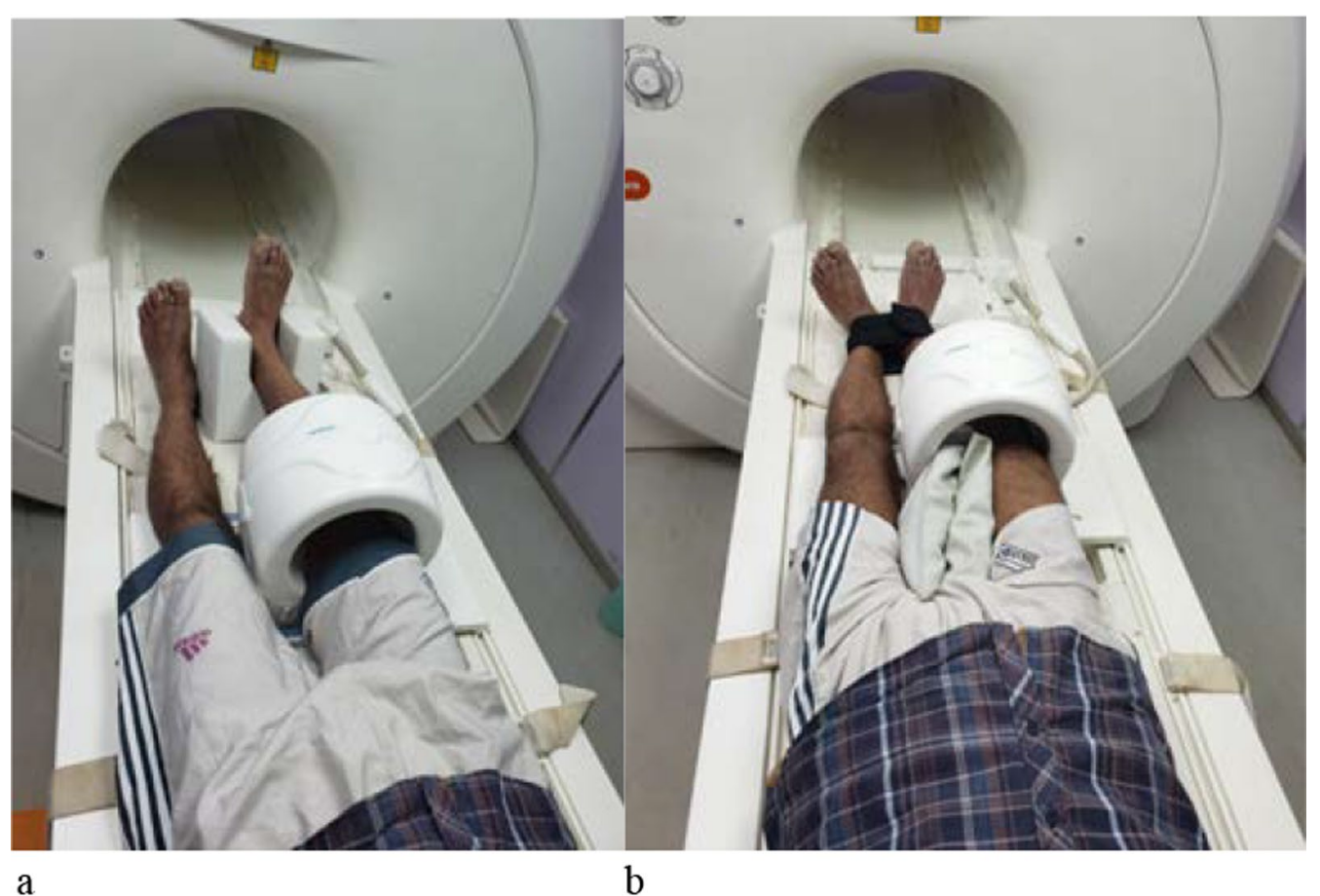

Fig. 2 Patient conventional position (pre-varus) (a). Varus stress position technique (b)

MMPRT can be diagnosed by the presence of the cleft/ truncation sign, giraffe neck sign, and medial meniscus extrusion. The cleft sign is described as a vertical fluid high-signal gap defect with a truncated meniscus seen at the root attachment on a posterior cut. The giraffe neck sign-also known as a meniscal body sign was determined by a lateral view of the giraffe neck-like shape of the medial meniscus (MM) posterior segment based on the coronal MR images. It was seen as a swollen/truncated slope-like shape of the MM posterior segment on the posterior coronal cut.

A feature of MME on the mid-coronal fluid-sensitive image was observed as an extra-articular partial or total displacement of the MM that extends $3 \mathrm{~mm}$ or more beyond the end margin of the medial tibial plateau. It was measured from the medial edge of the tibial plateau to the outer border of the MM. An MME of $\geq 3 \mathrm{~mm}$ is defined as a positive extrusion sign.

Furumatsu et al. [16] divided the MMPRT patients into three groups (acute group, subacute group, and chronic group) according to the duration from onset of injury or painful popping to MRI examination and according to MME measurement (Table 2).

The ghost meniscus sign, also called a white or empty meniscus sign, either partial or complete, was observed on the sagittal plane as an obliteration of a normally recognized meniscus or replacement of normal dark meniscal signal with a high-signal-intensity on the meniscal root. A partial tear was characterized by a partial abruption of the affected root, which is considered an abnormal signal intensity or morphology of the root, whereas a complete tear was characterized by complete root discontinuity.

Another sign of MMPRT seen on the axial view is the radial tear, which is described as a vertical high-signalintensity linear defect on the meniscal root.

The interpretation of MR images, including the presence of MME in pre- and post-varus positions, was assessed by two observers (A.A.Q., and H.E. with 7, and 33 years of experience, respectively). The consensus was reached by discussion in cases where disagreement was present.

\section{Statistical analysis}

Data entry and statistical analysis were performed using SPSS software version 22 (Statistical Package for Social Science) (SPSS Inc., Chicago, IL, USA). Continuous data were expressed as mean \pm standard deviation, whereas categorical data were expressed in frequencies and percentages. Quantitative variables were compared via one-way ANOVA (analysis of variance) to measure the MME among the three stages of chronicity, whereas quantitative variables in each stage were compared to measure the pre- and post-varus stress MME amount 


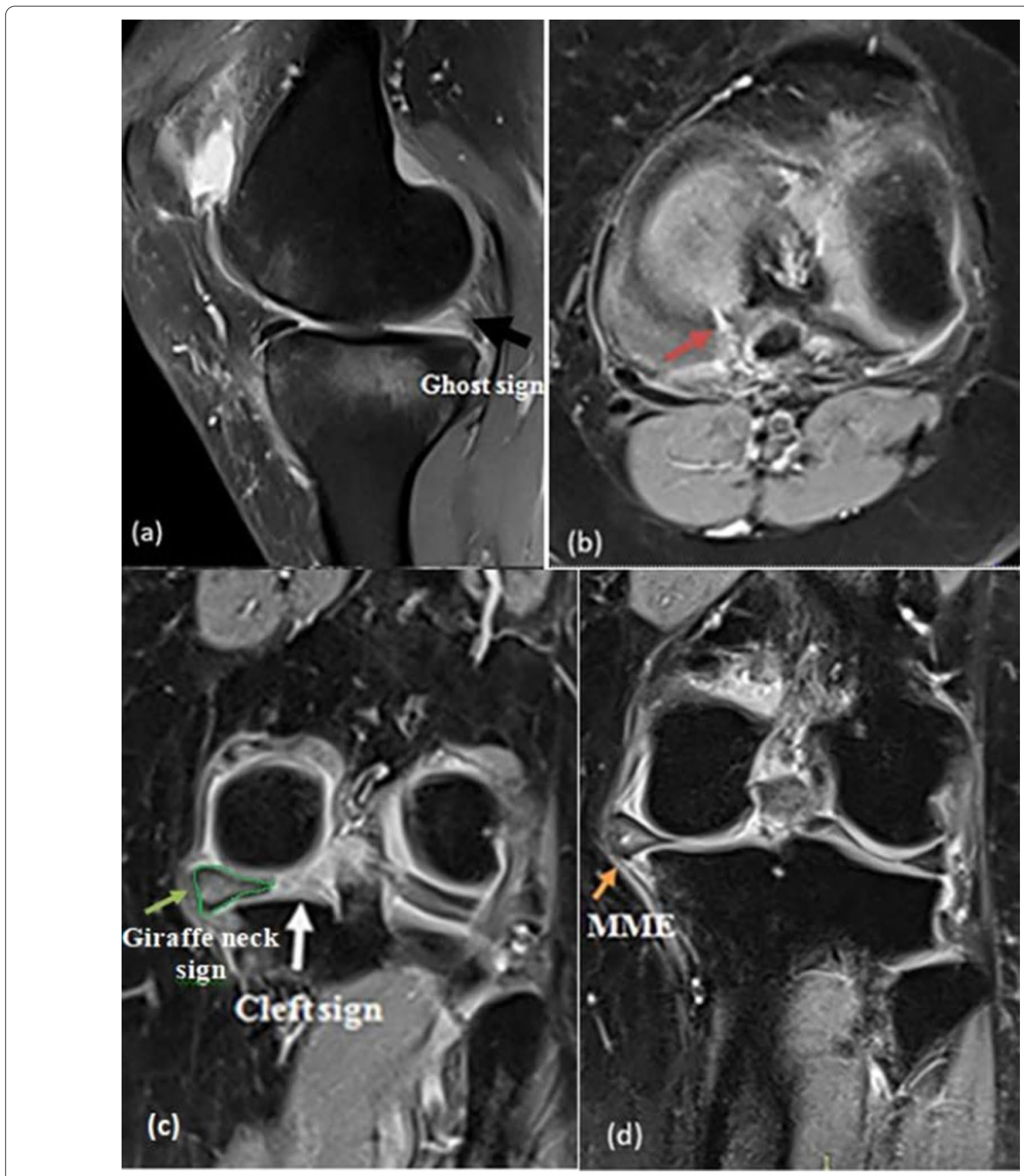

Fig. 3 Characteristic signs of the MMPRT on the MR images (PD-SPAIRWI). A 63-year-old woman (the left knee) showed: a A ghost "white meniscus" sign (black arrow) at the sagittal view. $\mathbf{b}$ A radial tear sign adjacent to the MM posterior root (red arrow) is seen as a linear fluid gap defect at axial view. c A giraffe neck sign (green line) with cleft or truncation sign (white arrow) at coronal view. d A medial meniscus extrusion (orange arrow) at coronal view

Table 2 Three groups of patients according to the onset and MME measurement of MMPRT

\begin{tabular}{llll}
\hline Variable & $\begin{array}{l}\text { Early } \\
<\mathbf{1} \text { month }\end{array}$ & $\begin{array}{l}\text { Subacute } \\
\mathbf{1}-\mathbf{3} \text { months }\end{array}$ & $\begin{array}{l}\text { Chronic } \\
\mathbf{4}-\mathbf{1 2} \text { months }\end{array}$ \\
\hline MME $(\mathrm{mm})$ & $3.0 \pm 1.2$ & $4.2 \pm 1.2$ & $5.8 \pm 1.6$ \\
\hline
\end{tabular}

using the paired sample t test. The qualitative variables in the pre- and post-varus stress technique between positive $\operatorname{MME}(\geq 3 \mathrm{~mm})$ and negative $\operatorname{MME}(<3 \mathrm{~mm})$ were compared using Fisher's exact test. MedCalc version 11.3 was used to compare the MRI findings of MMPRT with 
arthroscopy findings as a gold standard in 26 cases for the calculation of sensitivity, specificity, positive and negative predictive values, accuracy, and receiver operating characteristic (ROC) curve. A $P$ value of $<0.05$ was considered statistically significant.

\section{Results}

This study included 70 patients who presented with an episode of medial or posteromedial knee pain with or without popping sensation, wherein 14 patients were male $(20.0 \%)$ and 56 were female $(80.0 \%)$ with ages ranging from 24 to 70 years. Moreover, the mean age was $49.1 \pm 8.5$ years, and 38 patients $(54.3 \%)$ were older than 50 years. Concerning the symptoms, 46 patients (65.7\%) only presented with a popping sensation. Regarding the risk factors, the body mass index (BMI) ranged from 22.2 to $40.3 \mathrm{~kg} / \mathrm{m}^{2}$ with a mean of $31.7 \pm 4.95 \mathrm{~kg} / \mathrm{m}^{2}$, wherein overweight/obesity is considered a risk factor of MMPRT for those patients who had a BMI of $>30 \mathrm{~kg} / \mathrm{m}^{2}$, which comprised most cases $(42 / 70)$. On the other hand, it was found that 63 patients $(90.0 \%)$ had a history of osteoarthritis (OA), whereas 27 patients $(38.6 \%)$ had a history of trauma with or without OA.

According to duration onset or chronicity of MMPRT, it was found that 39 patients $(55.7 \%)$ were in the chronic stage, 16 patients $(22.9 \%)$ were in the acute stage, and 15 patients $(21.4 \%)$ were in the subacute stage.
Table 3 Comparison of pre and post-varus MME in 35 patients

\begin{tabular}{|c|c|c|c|c|c|}
\hline \multirow[t]{2}{*}{ MME } & \multicolumn{2}{|c|}{$\begin{array}{l}\text { Pre-varus MME } \\
(n=35)\end{array}$} & \multicolumn{2}{|c|}{$\begin{array}{l}\text { Post-varus MME } \\
(n=35)\end{array}$} & \multirow[t]{2}{*}{$P$ value } \\
\hline & No & $\%$ & No & $\%$ & \\
\hline Positive (MME $\geq 3$ ) & 22 & 62.9 & 35 & 100.0 & $0.000^{*}$ \\
\hline Negative $(\mathrm{MME}<3)$ & 13 & 37.1 & 0 & 0.0 & \\
\hline
\end{tabular}

Fisher Exact test was used to compare $M M E \geq 3 \mathrm{~mm}$ and $M M E<3 \mathrm{~mm}$ in pre and post-varus stress load technique

Table 4 MME Measurement according to chronicity

\begin{tabular}{lllll}
\hline & \multicolumn{2}{l}{ Chronicity } & & \multirow{2}{*}{ Pvalue $^{\mathbf{a}}$} \\
\cline { 2 - 4 } & Acute & Sub-acute & Chronic & \\
& Mean \pm SD & Mean \pm SD & Mean \pm SD & \\
\hline Pre-Varus & $2.89 \pm 0.47$ & $3.89 \pm 0.63$ & $4.72 \pm 1.71$ & $0.001^{*}$ \\
Post-Varus & $4.89 \pm 0.75$ & $6.07 \pm 1.16$ & $7.02 \pm 1.72$ & $0.001^{*}$ \\
P value $^{b}$ & $0.000^{*}$ & $0.000^{*}$ & $0.000^{*}$ & \\
\hline
\end{tabular}

${ }^{a}$ Comparison of MME measurements among the three types or stages of chronicity was done by using analysis of variance test (ANOVA test)

${ }^{b}$ Comparison between pre-varus and post-varus stress MME measurements in each separated stage was done by using (paired samples $T$ test)

\section{Characteristic MRI findings of MMPRT}

Of the 70 patients, 63 patients (90.0\%) exhibited positive MRI signs of MMPRT in the present study. The highest detection rate (97.1\%) was observed in the MME

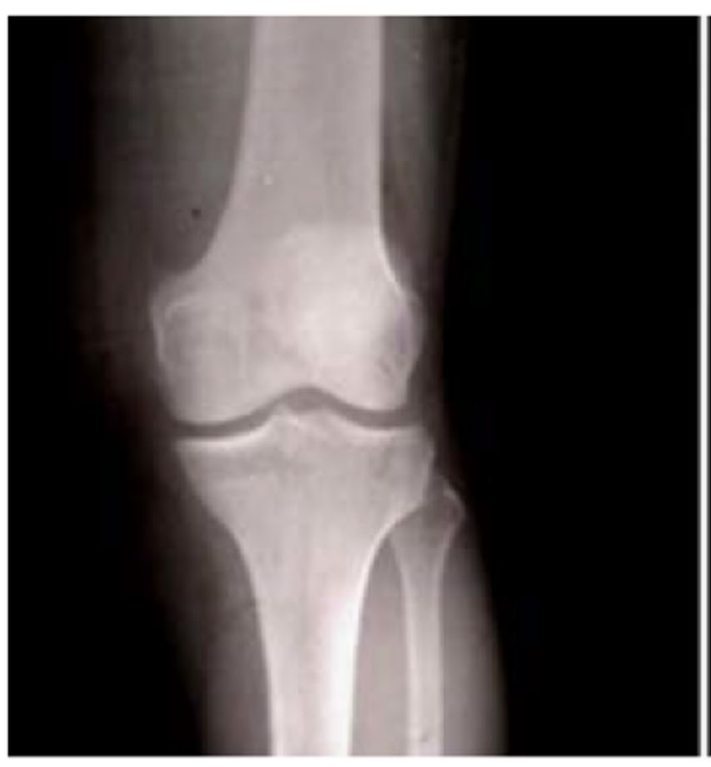

a

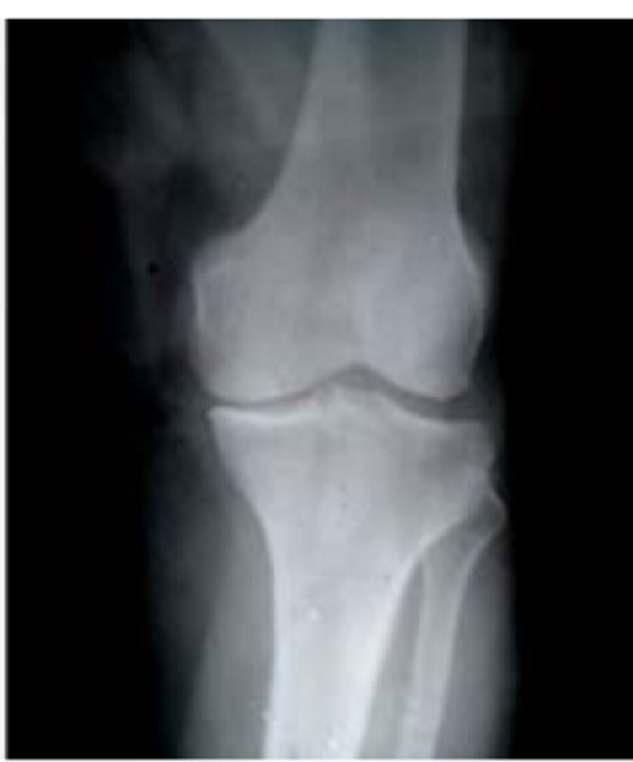

b

Fig. 4 A 47-year-old female patient, not obese $\left(\mathrm{BMl}=23.7 \mathrm{~kg} / \mathrm{m}^{2}\right)$, with a history of heavy labor work presented with a sudden onset of posteromedial left knee joint pain with a popping sound that occurred when she was doing housework for 5 months (chronic stage). At the time of injury, plain standing X-ray of the left knee looked normal (a); deteriorated knee joint space narrowing 5 months later on plain X-ray (b) revealed a sign of marked osteoarthritic changes in form of narrow medial joint compartment 

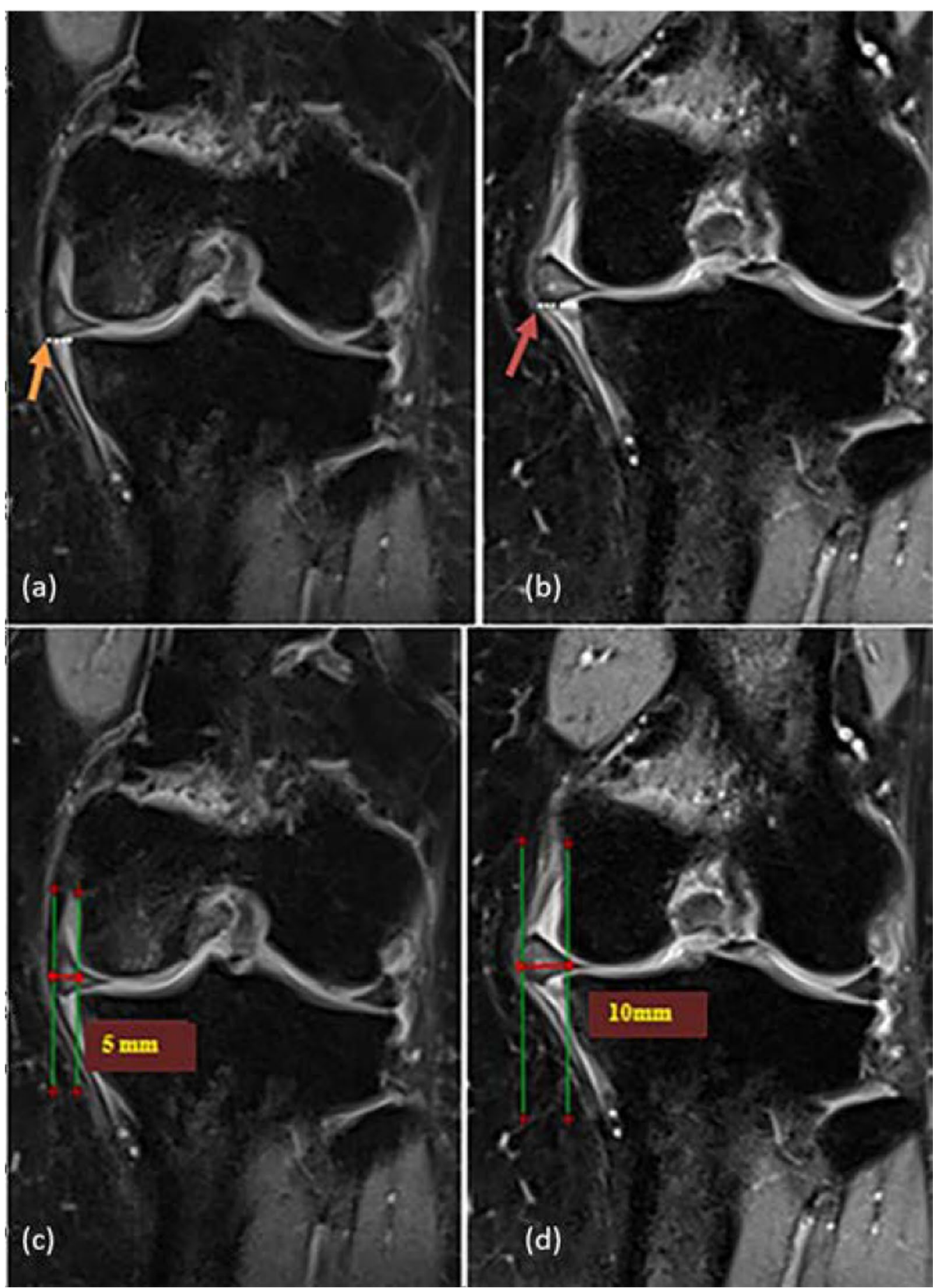

Fig. $5 \mathrm{MRl}$ of the same patient: comparison between the pre- and post-varus stress medial meniscus extrusion amount (MME) (c). Without varus stress position (left upper and left lower), the coronal PD-SPAIR WI of the left knee shows an extruded medial meniscus of approximately 5 mm (from the end of the medial tibial plateau to the end of the extruded meniscus) (orange arrow). With varus stress position (right upper and right lower), MME increased up to $10 \mathrm{~mm}$ from the end of the medial tibial plateau to the end of the extruded meniscus (red arrow)

sign. Overall, $88.6 \%$ of patients had the cleft sign, $87.1 \%$ had the ghost sign, $82.9 \%$ had the giraffe neck sign, and approximately two-thirds of them had the radial tear sign. In the current study, the coexistence of any two MRI signs of MMPRT, except for MME, also showed a high rate of approximately $90.0 \%$ which in turn could increase 
the diagnostic accuracy of MRI in diagnosing MMPRT. Furthermore, $54.3 \%$ of patients $(38 / 70)$ had a partial tear, $37.1 \%(26 / 70)$ had a complete tear, and only $8.6 \%(6 / 70)$ had no root tear. It was found that PD with fat saturation was more reliable than T2WI in the evaluation of MMPRT.

\section{Associated MRI findings in MMPRT}

Thirty percent of patients $(21 / 70)$ had associated anterior cruciate ligament (ACL) tear, 2.9\% (2/70) had posterior cruciate ligament (PCL) tear, and 54.3\% (38/70) presented with associated positive subchondral bone marrow edema.

\section{MRI technique with varus stress position}

It was found that the MRI technique with varus stress position could detect $100 \%$ of patients $(35 / 35)$ with positive MME $(\geq 3 \mathrm{~mm})$, whereas the MRI technique with the traditional position could detect only $62.9 \%$ of the cases $(22 / 35)$, indicating a highly statistically significant difference $(P<0.000)$ (Table 3$)$.

Regarding the chronicity of MMPRT, a significant increase in the amount of MME was observed in postvarus MRI compared with pre-varus MRI for each stage (acute, subacute, and chronic) $(P<0.000)$. The largest amount of MME was found in the chronic cases, followed by subacute and acute cases $(P<0.0001)$ (Table 4$)$.

Figures 4, 5, 6, and 7 show the representative cases for the pre- and post-varus MME comparison in chronic, subacute, and acute stages.

\section{Comparison of MRI findings with arthroscopic results in 26 patients with MMPRT}

A ROC analysis was performed to explain the performance of screening for the MMPRT via MRI in 26 patients at early and subacute stages with the gold standard arthroscopic results, while 44 patients were at chronic and subacute stages with some sequelae underwent high tibial osteotomy and conservative treatment. Overall, 20 were true positives, 1 was false positive, 4 were true negatives, and 1 was a false negative. The false-positive case was attributed to complex tear signs that masked the diagnosis of root tear, while the false-negative case had only one MRI sign which was not enough for diagnosis of
MMPRT. The sensitivity, specificity, accuracy, and positive and negative predictive values of MRI in the diagnosis of MMPRT patients were $95.24 \%, 80.0 \%, 92.31 \%$, $95.2 \%$, and $80.0 \%$, respectively, with an area under the curve of 0.876 .

\section{Discussion}

MRI is considered the examination modality of choice in the assessment of MMPRT through several characteristic radiological features [6].

To our knowledge, this is the first study to have developed a new MRI technique with varus stress position to address the challenges encountered by previous studies with regard to the measurement of the accurate degree of true MME in MMPRT in MRI scans at the supine position. This study included 70 patients who presented with an episode of medial or posteromedial knee pain with or without popping sensation.

According to the demographic data, the mean age of patients was $49.09 \pm 8.50$ years, with more predominance within female patients, and the ratio of women to men with MMPRT was 4:1. The mean BMI was $31.67 \pm 4.95 \mathrm{~kg} / \mathrm{m}^{2}$, and $60 \%$ of patients were overweight, and the majority of the patients $(55.7 \%)$ were in the chronic stage. This is consistent with the findings of Kim et al. [17], who found that MMPRT was more often observed in a chronic stage, especially in middle-aged or elderly female patients ( $>40$ years of age) with mild symptoms, degenerative changes, and commonly no clear history of trauma.

Regarding the risk factors of MMPRT, Hwang et al. [18] found that many factors may be related to MMPRTs, such as high BMI of $>30 \mathrm{~kg} / \mathrm{m}^{2}$, increased age, female gender, and varus malalignment. The results of this study were consistent with this finding, as it was found about $90 \%$ of cases had a history of osteoarthritis (OA), 54.3\% were elder than 50 years, the majority of patients $(80 \%)$ were female gender, and $58.6 \%$ were obese.

Based on the patients' clinical presentation, approximately $65.7 \%$ of the studied patients presented with posterior knee pain associated with popping sensation, which was a good indicator in the diagnosis. This is in line with the study by LaPrade et al. [19]] and Lee et al. [20] which revealed that the remember of time of painful popping sounds is so important to identify the duration

\footnotetext{
(See figure on next page.)

Fig. 6 A 46-year-old female homemaker patient, obese $\left(B M I=41.4 \mathrm{~kg} / \mathrm{m}^{2}\right)$, presented with significant posteromedial left knee pain with a popping sound for 7 weeks (subacute stage) after stepping into a hole. By the comparison between the pre- and post-varus stress medial meniscus extrusion amount (MME). Without varus stress position (a and $\mathbf{c}$ ), the coronal PD-SPAIR WI of the left knee shows an extruded medial meniscus of approximately $3.4 \mathrm{~mm}$ from the medial margin of the tibial plateau to the outer border of the extruded meniscus (orange arrow). With varus stress position (b and $\mathbf{d}$ ), shows an increased MME amount of approximately $6.8 \mathrm{~mm}$ (red arrow)
} 


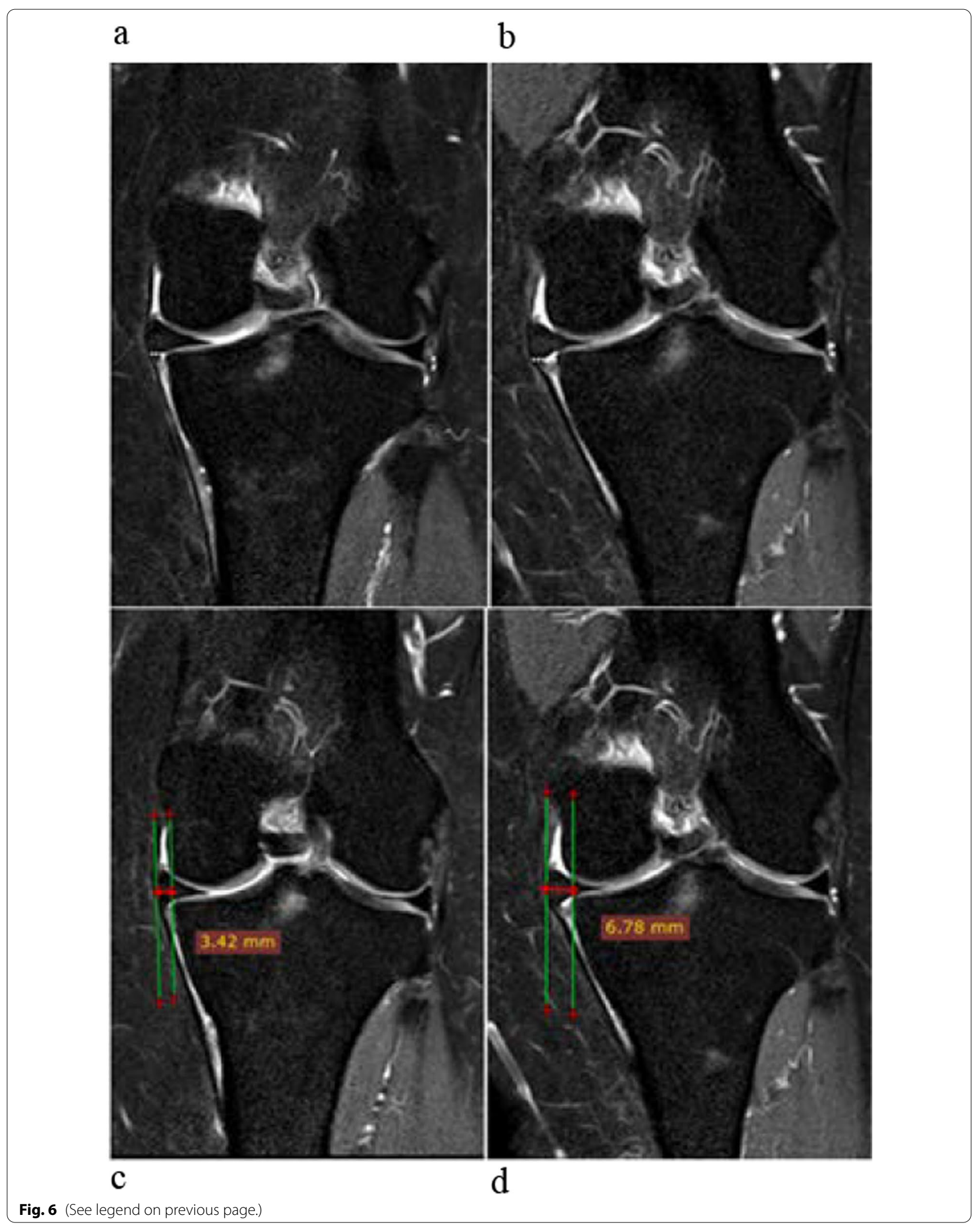


of onset and treatment plan of MMPRT. On the other hand, it has not been investigated whether a single event of painful popping is a typical clinical symptom of other meniscal tears.

The diagnosis of meniscal root tears is considerably difficult, with a reported detection rate of $73 \%$ to $89 \%$, even if it is based on characteristic MRI findings [2, 21]. The current results indicated that approximately $90.0 \%$ of the studied patients had positive MRI signs of MMPRT.

The evaluation of MRI and arthroscopic procedures is essential to reach a proper definite MMPRT diagnosis. In this study, MRI showed $95.24 \%$ sensitivity, $80.0 \%$ specificity, $95.2 \%$ positive predictive value, $80.0 \%$ negative predictive value, and $92.31 \%$ accuracy in diagnosing MMPRT. This is consistent with a systematic review published by Oei et al. [22], who concluded that MRI demonstrates sensitivity and specificity rates of $93 \%$ and $88 \%$, respectively, considering arthroscopy being the gold standard reference in the diagnosis of a medial meniscal tear.

In the present study, several characteristic MR findings have indicated the presence of MMPRT. It was reported that the highest detection rate of approximately $97.1 \%$ was observed in the MME sign. However, Choi et al. [13] reported a lower detection rate of MRI-based MME $(\geq 3 \mathrm{~mm})$ in MMPRT patients $(83.3 \%)$. The higher detection rate in the current study was attributed to the varus stress position. Overall, $88.6 \%$ of the studied patients had the cleft sign, $82.9 \%$ had the giraffe neck sign, $87.1 \%$ had ghost sign, and $65.7 \%$ had radial tear sign. The results of this study are consistent with those of another similar study conducted by Furumatsu et al. [14] who illustrated that the cleft, ghost, giraffe neck, and radial tear signs were detected in $81.7 \%, 80 \%, 81.7 \%$, and $69.6 \%$ of the MMPRT MRI images, respectively. Although the medial extrusion sign was most commonly observed in MMPRT (90\%), it was found to be an indirect sign and not specific for meniscal root tears. Furumatsu et al. [14] detected that the coexistence of any two characteristic MRI signs, except for the medial extrusion sign, was observed in $91.7 \%$ of MMPRT patients. This result is consistent with that of the present study, which indicated a high detection rate of approximately $90.0 \%$.

Although the presence of extruded meniscus of more than $3 \mathrm{~mm}$ was considered an indirect sign in meniscal root tears as reported by Bhatia et al. [11], it could be utilized as a further guide in MMPRT diagnosis as recommended by Koo et al. [15].

In the present study, MRI technique with varus stress position was performed on 35 patients. In this new technique, we measured the true MME amount in MMPRT patients as they were exposed to postural load caused by the stress varus position technique that was relatively simulating weight-bearing MRI and standing plain X-ray.

Moreover, it was observed that approximately two-thirds of studied patients $(62.9 \%)$ had positive MME $(\mathrm{MME} \geq 3)$ at pre-varus assessment, whereas all patients (100.0\%) showed positive MME at post-varus assessment, with a statistically significant difference between pre-varus MME $(3.75 \pm 1.30 \mathrm{~mm})$ and post-varus MME $(5.89 \pm 1.52 \mathrm{~mm})$. This finding is consistent with that of a study by Lerer et al. [23] who found meniscus extrusion could be considered a diagnostic sign for MMPRT in MRI. According to the duration of onset or the chronicity of MMPRT, the MME measurements in the present study illustrated that the degree or amount of MME was highest in the chronic cases, followed by the subacute and acute cases, with a statistically significant difference in both the pre and post-stress varus position techniques.

In the comparison between pre- and post-varus stress amount of MME in all three stages of MMPRT, it was found that statistically significant differences were observed between pre- and post-varus MME amount in each stage of chronicity. The findings of Furumatsu et al. [14] are also consistent with pre-varus stress results of the MME measurement in the current study. One of the positive findings of the present study was mentioned by Chung et al. [24], who found that decreased MME $(3.5 \pm 1.4 \mathrm{~mm})$ at 1 year after arthroscopic repairs of MMPRT had more appropriate clinical scores and radiographic features at 5-year follow-up compared with increased MME $(5.1 \pm 1.4 \mathrm{~mm})$ at 1 year after MMPRT pullout repairs.

Concerning MRI-based associated findings of MMPRT, the current study showed that $30.0 \%$ of the studied patients of MMPRT had associated ACL tear, whereas only $2.9 \%$ of them had PCL tear, which is consistent with the findings of Lee et al. [25] and Ra et al. [26], who stated that acute medial meniscal root tears could occur as a result of multiligamentous injuries in the knee joint, such as ACL and PCL tears.

\footnotetext{
(See figure on next page.)

Fig. 7 A 54-year-old female employee, obese $\left(B M I=39.6 \mathrm{~kg} / \mathrm{m}^{2}\right)$, presented with a sudden onset of posteromedial right knee pain with a popping sound after a twisting injury. The patient had difficulty climbing the stairs and squatting for one week (acute or early period). By the comparison between the pre- and post-varus stress medial meniscus extrusion amount (MME). Without varus stress position (a and c), the coronal PD-SPAIR WI of the right knee shows a negative extruded medial meniscus of approximately $2.5 \mathrm{~mm}$ from the medial margin of the tibial plateau to the outer border of the extruded meniscus (orange arrow). With varus stress position ( $\mathbf{b}$ and $\mathbf{d}$ ), the MME changed from negative to positive extrusion of approximately $4.5 \mathrm{~mm}$ (white arrow)
} 


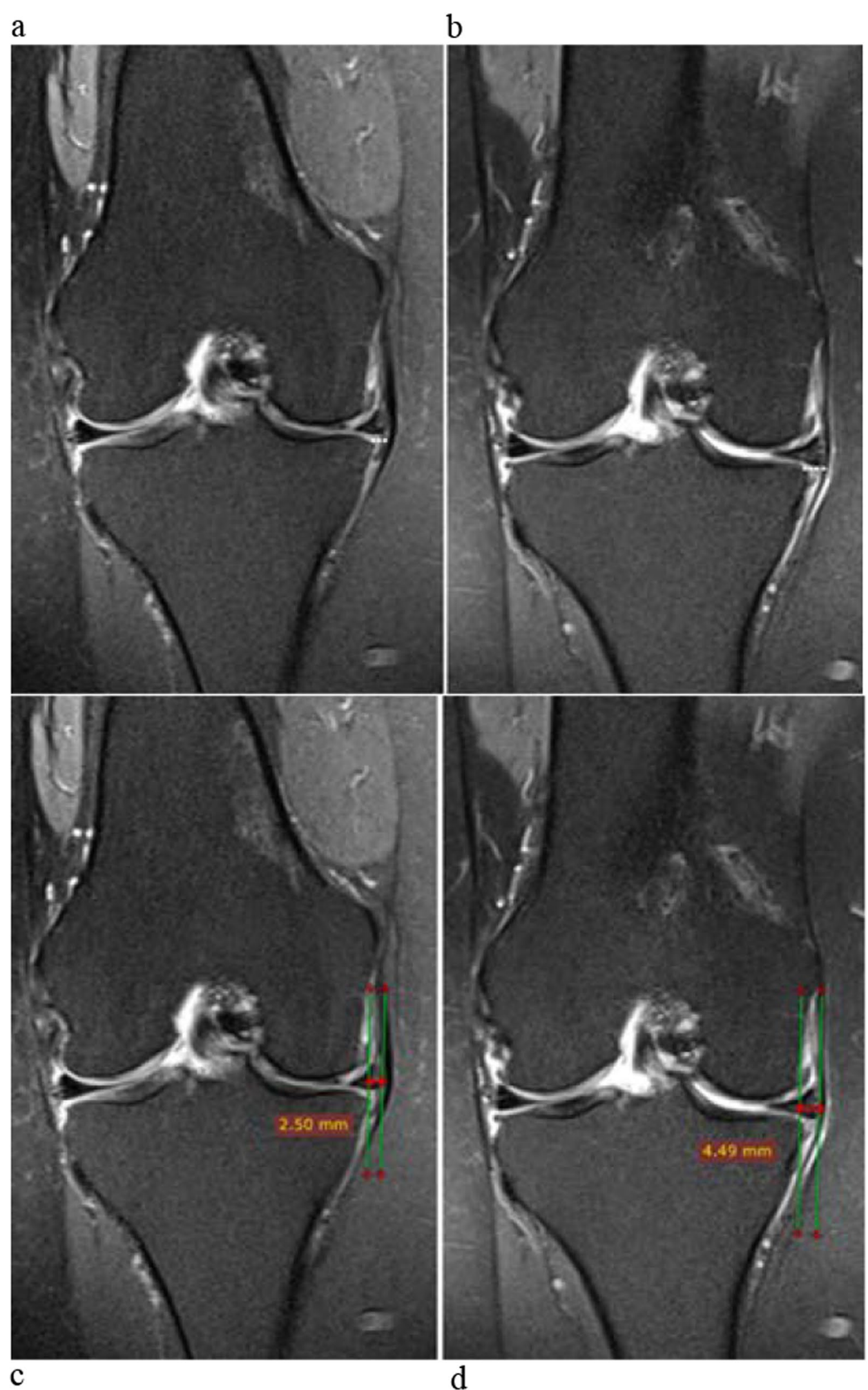

Fig. 7 (See legend on previous page.) 
On the other hand, $54.3 \%$ of patients showed positive subchondral bone marrow edema based on current results, which is consistent with the findings of a previous study by Bhatia et al. [11], who found that ipsilateral tibiofemoral compartment bone marrow edema is more common when MMPRT is present.

Regarding the treatment of MMPRTs, the current results showed that the majority of patients underwent valgus HTO, only $10.0 \%$ in the acute stage underwent arthroscopy, 27.1\% underwent both valgus HTO and meniscal arthroscopic repair, and $20 \%$ underwent conservative therapy. That is why only a small part of the study group had an arthroscopic reference standard. This finding is consistent with that of Chung et al. [27], who demonstrated that in the case of MMPRT associated with varus misalignment, osteotomy should be performed either alone or with another surgical intervention.

The present study has strength points, including the prospective design, confirmatory arthroscopic reference, and information on the new MRI technique with varus stress position in measuring the true MME amount that could solve the problem related to inadequate extrusion measurement performed via supine non-weight-bearing MRI.

However, this study has several limitations, such as the limited number of cases that are screened via MRI and confirmed arthroscopically because the majority of the cases in the current study were in the chronic stage who underwent either osteotomy or conservative treatment, as well as the unavailability of weight-bearing upright MRI to compare it with the results of MME measurements performed using the MRI technique with varus stress.

\section{Conclusion}

MRI has been proven to be highly accurate in detecting MMPRT. Therefore, it is considered the imaging of choice technique for diagnosing MMPRT through several direct and indirect characteristic MRI signs. The new MRI technique with varus stress position has shown clinical importance in assessing the presence of MME in non-extruded pre-varus MRI and in measuring the accurate degree of severity of true extruded meniscus in MMPRT. The final goal of this study is to help orthopedic surgeons choose the most favorable treatment strategy for MMPRT based on the duration or chronicity of the root tear, associated risk factors, and/or the accurate degree of severity of true MME.

\section{Abbreviations}

ACL: Anterior cruciate ligament; BMI: Basal metabolic rate; FS: Fat-saturation; FSE: Fast spin-echo; HTO: High-tibial osteotomy; MM: Medial meniscus; MMPRT: Medial meniscal posterior root tear; MRI: Magnetic resonance imaging; PCL: Posterior cruciate ligament; PD: Proton density; ROC: Receiver operator characteristic.
Acknowledgements

The authors thank all the study participants for their patience and support.

Authors' contributions

HE suggested and developed the research idea and H.E.G. established the technical aspects. HE and AAQ were responsible for preparing cases, reporting them, performing required measurements, and preparing figures and tables. MA performed statistical analysis, AAQ, AAH, HAK, HEG, MA, and NAA were responsible for writing and revising the manuscript, All authors have a major contribution in preparing and editing the manuscript. All authors read and approved the final manuscript.

\section{Funding \\ No.}

\section{Availability of data and materials}

The data that support the findings of this study are available from Orthopaedic and Radiology departments-Assiut University, but restrictions apply to the availability of data, which are used under license for this study, and so were not publicly available. Data were available from authors upon request with permission of the head of the Radiology department-Assiut University.

\section{Declarations}

Ethics approval and consent to participate

This study had approval from Egypt, Assiut University, Faculty of Medicine Research Ethics Committee (IRB Number 17200240).

\section{Ethical consideration}

(1) Risk Benefit assessment for all patients was indicated for the examination during this study. (2) Confidentiality: All patients' data were confidentially kept.

(3) The research done by scientifically qualified and trained personnel only.

\section{Informed written consents}

Obtained.

\section{Consent for publication}

Not applicable.

\section{Competing interests}

Authors declare that they had no competing interests.

\section{Author details}

${ }^{1}$ Department of Diagnostic Radiology, Faculty of Medicine, Assiut University, Assiut 71515, Egypt. ${ }^{2}$ Department of Orthopaedic, Faculty of Medicine, Assiut University, Assiut, Egypt.

Received: 8 December 2021 Accepted: 1 February 2022

Published online: 08 February 2022

\section{References}

1. Baker JC, Friedman MV, Rubin DA (2018) Imaging the postoperative knee meniscus: an evidence-based review. Am J Roentgenol 211(3):519-527

2. Palisch AR, Winters RR, Willis MH et al (2016) Posterior root meniscal tears: preoperative, intraoperative, and postoperative imaging for transtibial pullout repair. Radiographics 36:1792-1806

3. Pache S, Aman ZS, Kennedy M et al (2018) Meniscal roots: current concepts review. Arch Bone Jt Surg 6:250-259

4. Bae JH, Paik NH, Park GW et al (2013) Predictive value of painful popping for a posterior root tear of the medial meniscus in middle-aged to older Asian patients. Arthrosc J Arthrosc Relat Surg 29:545-549

5. Ramnath RR, Magee T, Wasudev N, Murrah R (2006) Accuracy of 3-T MRI using fast spin-echo technique to detect meniscal tears of the knee. Am J Roentgenol 187:221-225

6. Ozkoc G, Circi E, Gonc U et al (2008) Radial tears in the root of the posterior horn of the medial meniscus. Knee Surg SportsTraumatol Arthrosc 16:849-854 
7. Faucett SC, Geisler BP, Chahla J et al (2019) Meniscus root repair vs meniscectomy or nonoperative management to prevent knee osteoarthritis after medial meniscus root tears: clinical and economic effectiveness. Am J Sports Med 47(3):762-769

8. Koenig JH, Ranawat AS, Umans HR, DiFelice GS (2009) Meniscal root tears: diagnosis and treatment. Arthroscopy 25:1025-1032

9. Doherty DB, Lowe WR (2016) Meniscal root tears: identification and repair. Am J Orthop (Belle Mead NJ) 45:183-187

10. Hein CN, Deperio JG, Ehrensberger MT, Marzo JM (2011) Effects of medial meniscal posterior horn avulsion and repair on meniscal displacement. Knee 18:189-192

11. Bhatia S, Laprade CM, Ellman MB, Laprade RF (2014) Meniscal root tears: significance, diagnosis, and treatment. Am J Sports Med 42:3016-3030

12. Furumatsu T, Fujii M, Kodama Y, Ozaki T (2017) A giraffe neck sign of the medial meniscus: a characteristic finding of the medial meniscus posterior root tear on magnetic resonance imaging. J Orthop Sci 22:731-736

13. Choi CJ, Choi YJ, Song IB, Choi CH (2011) Characteristics of radial tears in the posterior horn of the medial meniscus compared to horizontal tears. Clin Orthop Surg 3:128

14. Furumatsu T, Kamatsuki Y, Fujii M et al (2017) Medial meniscus extrusion correlates with disease duration of the sudden symptomatic medial meniscus posterior root tear. OrthopTraumatol Surg Res 103:1179-1182

15. Koo JH, Choi S-H, Lee SA, Wang JH (2015) Comparison of medial and lateral meniscus root tears. PLOS ONE 10:e0141021

16. Hussain ZB, Chahla J, Mandelbaum BR et al (2019) The role of meniscal tears in spontaneous osteonecrosis of the knee: asystematic review of suspected etiology and a call to revisit nomenclature. Am J Sports Med 47:501-507

17. Kim SB, Ha JK, Lee SW et al (2011) Medial meniscus root tear refixation: comparison of clinical, radiologic, and arthroscopic findings with medial meniscectomy. Arthrosc J Arthrosc Relat Surg 27:346-354

18. Hwang BY, Kim SJ, Lee SW et al (2012) Risk factors for medial meniscus posterior root tear. Am J Sports Med 40:1606-1610

19. Laprade CM, Foad A, Smith SD et al (2015) Biomechanical consequences of a nonanatomic posterior medial meniscal root repair. Am J Sports Med 43:912-920

20. Lee DW, Ha JK, Kim JG (2014) Medial meniscus posterior root tear: a comprehensive review. Knee Surg Relat Res 26:125-134

21. Moatshe G, Chahla J, Slette E et al (2016) Posterior meniscal root injuries: a comprehensive review from anatomy to surgical treatment. Acta Orthop 87:452-458

22. Oei EHG, Nikken JJ, Verstijnen ACM et al (2003) MR imaging of the menisci and cruciate ligaments: a systematic review. Radiology 226:837-848

23. Lerer DB, Umans HR, Xu MX, Jones MH (2004) The role of meniscal root pathology and radial meniscal tear in medial meniscal extrusion. Skeletal Radiol 33:569-574

24. Chung KS, Ha JK, Ra HJ et al (2017) Pullout fixation of posterior medial meniscus root tears. Am J Sports Med 45:42-49

25. Lee DH, Lee BS, Kim JM et al (2011) Predictors of degenerative medial meniscus extrusion: radial component and knee osteoarthritis. Knee Surg Sports Traumatol Arthrosc 19:222-229

26. Ra HJ, Ha JK, Jang HS, Kim JG (2015) Traumatic posterior root tear of the medial meniscus in patients with severe medial instability of the knee. Knee Surg Sports Traumatol Arthrosc 23:3121-3126

27. Chung KS, Ha JK, Yeom CH et al (2015) Comparison of clinical and radiologic results between partial meniscectomy and refixation of medial meniscus posterior root tears: aminimum5-year follow-up. Arthrosc J Arthrosc Relat Surg 31:1941-1950

\section{Publisher's Note}

Springer Nature remains neutral with regard to jurisdictional claims in published maps and institutional affiliations.

\section{Submit your manuscript to a SpringerOpen ${ }^{\circ}$ journal and benefit from:}

- Convenient online submission

- Rigorous peer review

- Open access: articles freely available online

- High visibility within the field

- Retaining the copyright to your article

Submit your next manuscript at $\boldsymbol{\nabla}$ springeropen.com 